EP-96

\title{
The effect of synbiotic drink from kefir milk and jicama concentrate (Pachyrhizus erosus) on malondialdehyde level and superoxide dismutase activity in the liver tissue of the hyperlipidemic rats (Rattus norvegicus)
}

\author{
Rafik PRABOWO$^{* 1}$, Saosanti MARIANA ${ }^{2}$ \\ ${ }^{1}$ Clinical Program of Medicine, Faculty of Medicine, Universitas Islam Indonesia, Indonesia \\ ${ }^{2}$ Department of Chemical Engineering, Faculty of Industrial Technology, Universitas Islam Indonesia, Indonesia
}

Introduction: The aim of this research is to know the effect of synbiotic drinks from kefir milk and jicama concentrate on MDA level and SOD activity in the liver tissue of the hyperlipidemic rats.

Methods: This research used 25 rats divided into 5 groups (K+, K-, P1, P2, and P3). Group of K+, P1, P2, P3 were given quail egg yolk for the first 4 weeks. For the next 4 weeks, K+ and K- group were only given fed ad libitum. Group of P1, P2, and P3 were given synbiotic with the formulation of P1: 85\% kefir milk (K) and 15\% jicama concentrate (J), P2: 75\% K, 25\% J, and P3: 65\% K, 35\% J. The dose of quail egg yolks and synbiotic was $5 \mathrm{~mL} / 200 \mathrm{grBW}$. In the end, the animal model was terminated to get liver organs to measure the MDA level and SOD activity.

Results: Mean of MDA level (nmol/gr) were $11.8 \pm 0.17$ (K+), $2.5 \pm 0.12$ (K-), $7.7 \pm 0.18$ (P1) $5.7 \pm 0.10$ (P2), $4.1 \pm 0.09$ (P3). The result showed significant differences between all groups $(p<0.001)$. Mean of SOD activity (\%) were $21.43 \pm 2.52(\mathrm{~K}+), 71.43 \pm 3.91(\mathrm{~K}$-), 30.71 \pm 1.53 (P1) $50.35 \pm 2.84$ (P2), $63.93 \pm 1.53$ (P3). The result showed significant differences between all groups $(p<0.001)$ except between $\mathrm{K}+$ with $\mathrm{P} 1$ and $\mathrm{K}$.

Conclusions: Intervention of synbiotic drink from kefir milk and jicama concentrate significantly decrease MDA level in all groups and increase SOD activity in the P2 and P3 groups, with P3 group clinically significant. 